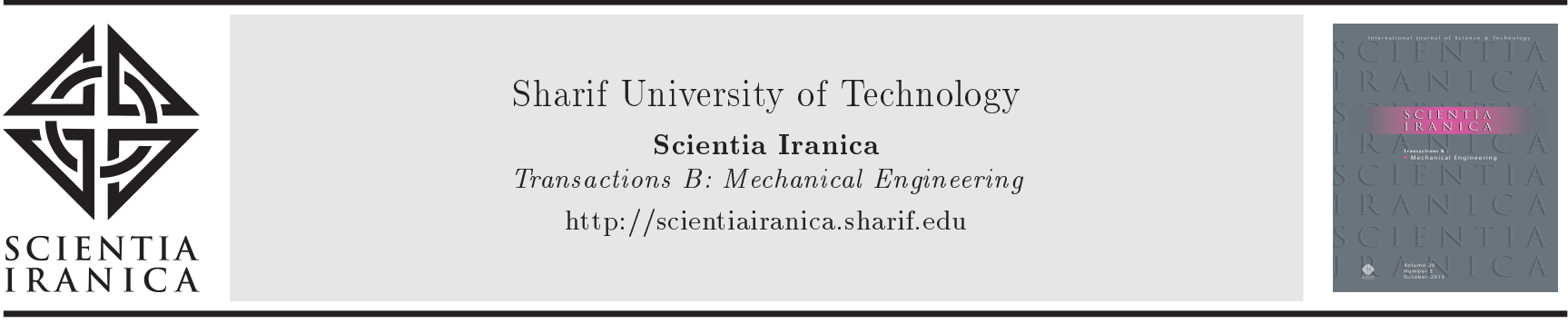

\title{
Analytical model of the influence of recirculation on premixed combustion of lycopodium dust particles: A study of thermal resistance and random distribution of particles
}

\author{
M. Bidabadi, M. Harati*, and H. Moghadasi \\ School of Mechanical Engineering, Department of Energy Conversion, Iran University of Science and Technology (IUST), Narmak, \\ Tehran, 16846-13114, Iran.
}

Received 21 October 2017; received in revised form 16 September 2018; accepted 17 June 2019

\author{
KEYWORDS \\ Organic dust particle; \\ Thermal resistance; \\ Heat loss; \\ Random combustion; \\ Heat recirculation.
}

\begin{abstract}
In the present study, a comprehensive analytical method was developed to model flame propagation through organic particles with air as a two-phase mixture, considering random distribution and particles thermal resistance. For this purpose, the structure of flame contained a preheat-vaporization zone, a reaction zone where vaporization and convection rates of particles were negligible, and a post flame zone where diffusive terms were negligible in comparison with the zone for other terms. In order to enhance combustion efficiency, the exhausted heat from the post flame zone was recirculated back to the preheat zone. Since the stream consisted in a high-temperature gaseous mixture, it could enhance the temperature of the initial two-phase mixture entering the combustion chamber. A reasonable agreement between the results of the analytical approach and the experimental findings was obtained. In addition to the random distribution of particles and heat recirculation phenomenon, the effect of thermal resistance on the combustion properties such as burning velocity and flame temperature was studied through non-zero Biot numbers in the proposed model. Moreover, the variations of several parameters including equivalence ratio, particle diameter, and Lewis number were investigated.
\end{abstract}

(C) 2020 Sharif University of Technology. All rights reserved.

\section{Introduction}

Dust cloud explosion has been a serious threat to human being and their properties for the last 150 years. There is a high risk of explosion when combustible dust is suspended in the air in particular circumstances. This phenomenon is common and can cause high costs

\footnotetext{
*. Corresponding author. Tel.: +9821 73228918 E-mail addresses: bidabadi@iust.ac.ir (M. Bidabadi); harati@mecheng.iust.ac.ir (M. Harati); hesam_moghadasi@mecheng.iust.ac.ir (H. Moghadasi)
}

doi: $10.24200 /$ sci. 2019.5423 .1264 in a wide range of industries. Therefore, proper understanding of the origin, prevention, and mitigation of dust explosions is vital. Several materials trigger this event such as coal, peat, and metals [1-3]. Recent developments in technology and industries have led to a growing need for applicable energy. Biomass has been identified as a renewable energy resource and a good candidate for alternative fuel [4-6]. In this mathematical work, lycopodium is considered as a volatile fuel, especially due to its appreciable flammability and dispensability. Lycopodium has been known as a reference particle in organic dust combustion research since being mono-size is a fundamental supposition in analytical models. Moreover, the amount of unfavor- 
able residues produced by lycopodium during combustion is unimportant. A lycopodium particle generally contains about $50 \%$ fat oil, $24 \%$ sporopollenin, and $2 \%$ sucrose. In combustion processes, the particle misses some part of its mass during flame propagation since lycopodium is a volatile material $[7,8]$. Within the last few years, many studies have been conducted regarding combustion of organic dust particles. Bidabadi et al. [9] proposed a novel mathematical model for the combustion of micro-organic dust particles. In their study, the effects of random combustion, Lewis number, and size of particles on the combustion properties of biofuel particles such as burning velocity, flame temperature, and effective equivalence ratio were investigated. Mostafavi et al. [10] carried out experimental and mathematical modeling of lycopodium dust particles. An extensive research was conducted by Proust [11] on the initiation and propagation of flame in dust clouds to provide a deeper understanding of the incidence of thermal radiation and turbulence in dust explosion. In this regard, the laminar flame speeds and flame temperatures were measured for several types of combustible dust-air mixtures [12]. Following this research with higher emphasis on the lycopodium as a combustible dust particle, Han et al. [13] empirically analyzed the flame propagation mechanism and the behavior of dust particles in a vertical duct. Some parametric studies were conducted by Daou [14] to realize the influence of heat loss, preferential diffusion, and reversibility of reaction. Until 2009, there was no research addressing the recirculation effect on dust particles. Bidabadi et al. [15] developed a comprehensive model to consider this important dimension. Besides, Bidabadi and Rahbari [16] studied the influence of temperature alteration between particle and gas in a combustible mixture with homogeneous distribution of volatile fuel particles. In another study, Rockwell and Rangwala [17] scrutinized a premixed dust-air flame in the presence of a homogeneous gas-phase reaction front. Moghadasi et al. [18] studied combustion of organic particles in a counterflow arrangement considering porosity and thermal radiation effects. Soltaninejad et al. [19] analyzed thermal resistance of the micro-organic dust combustion cogitating particles. They presumed that gaseous fuel mixture was formed as a result of particle pyrolysis. Reviewing the existing literature indicates that few attempts have been made to numerically determine the non-uniform distribution of combustible dust-air mixture. This study reflects the combustion of microorganic particles considering random distribution and thermal resistance of particles. The particles were dispersed in the preheat zone, arbitrarily, presuming that the fuel particles were initially volatilized to yield a gaseous fuel. The flame structure was separated into three zones as exhibited in Figure 1. The first region was a preheat-vaporization zone where the chemical reaction rate was neglected. The second one was a reaction zone where the volatile fuel particles were combusted in the gaseous phase. In this zone, the vaporization rate and convection terms were ignorable in order of magnitude. The third zone was a post-flame one in which chemical reaction and vaporizations rates were supposed to be smaller than other terms. The exhaust heat recirculation from the post-flame zone to the preheat zone (leading to enhancement in the combustion performance of the process) was modeled in this research. The role of thermal resistance of the particles was incorporated into the governing equations via the dimensionless Biot number $(\mathrm{Bi}=h r / \lambda)$. The Biot number states the easiness of heat flow through the interface of the powder deposit and its surrounding, relating to the easiness of heat conduction through the powder. The features of flame propagation and the structure of combustion zone with the impact of non-unity Lewis number and heat recirculation were mathematically explored in order to elucidate the flame propagation mechanisms in the organic dust clouds. Evaluating the accuracy of the present model was car-

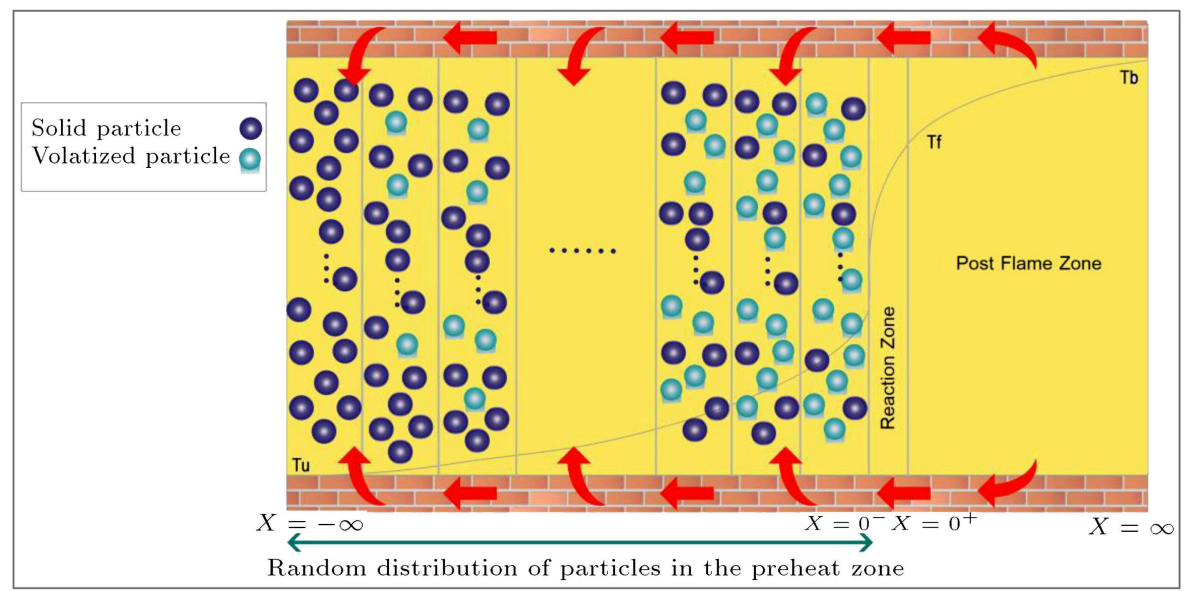

Figure 1. Schematic view of the flame propagation mechanism and heat recirculation. 
ried out by comparing the burning velocity obtained by the developed model with the empirical and analytical data $[11,20,21]$.

\section{Theoretical analysis}

In this study, it is assumed that the fuel particles are first volatilized to shape an identified gaseous compound. The vaporization kinetic is formulated as [21]:

$$
\omega_{v}=\exp (-\mathrm{Bi}) A n_{s} 4 \pi r^{2}\left(T-T_{u}\right)^{n},
$$

where $\omega_{v}$ is the rate of vaporization of fuel particles. The constant quantities $A$ and $n$ are considered to be known. One-step overall reaction is used to model the combustion process as:

$$
\nu_{F}[F]+\nu_{O_{2}}\left[O_{2}\right] \rightarrow \nu_{P}[P],
$$

where $F, O_{2}$, and $P$ signify the fuel, oxygen, and product and $v_{F}, V_{O_{2}}$, and $v_{P}$ are their stoichiometric coefficients. The parameter of Zeldovich number is obtained by:

$$
\mathrm{Ze}=\frac{E_{a}\left(T_{f}-T_{u}\right)}{R T_{f}^{2}},
$$

where $E_{a}, T_{f}, T_{u}$, and $R$ are the activation energy, flame temperature, fresh mixture temperature, and universal gas constant, respectively.

Mass and energy conservation equations are presented in Eqs. (4) and (5), respectively.

- Mass conservation equation:

$$
\rho v=\text { const, }
$$

- Energy conservation equation:

$$
\begin{aligned}
\rho v C \frac{d T}{d x}= & \lambda_{u} \frac{d^{2} T}{d x^{2}}+\omega_{F} \frac{\rho_{u}}{\rho} Q \\
& -\left(\omega_{v} \frac{\rho_{u}}{\rho} Q_{v}+\operatorname{sign}(x) \frac{\rho_{u}}{\rho} Q_{L}\right),
\end{aligned}
$$

where:

$$
\begin{aligned}
& \omega_{F}=\nu_{F} W_{F} C_{F} k_{r}, \\
& k_{r}=B_{r} \exp \left(-\frac{E_{a}}{R T_{f}}\right),
\end{aligned}
$$

where $Q_{v}$ is the amount of heat required for vaporizing the solid particles and $Q_{L}$ is the amount of heat exchanged between the gas mixture and its surrounding estimated by:

$$
Q_{L}=k_{2}\left(T-T_{W}\right) .
$$

In the above equation, $T, T_{W}$, and $k_{2}$ denote the mixture temperature, wall temperature, and the ratio of convective heat transfer coefficient to chamber width. The total heat capacity of mixture can be outlined as follows [21]:

$$
C=C_{P}+\left(\frac{4}{3} \pi R^{3}\right) \frac{C_{s} \rho_{s} n_{s}}{\rho} .
$$

In which $C_{P}$ and $C_{s}$ are the heat capacities of gaseous phase in the mixture and solid particles, respectively. The dimensionless parameters are implemented in the governing equations as:

$$
\begin{aligned}
& \theta=\frac{T-T_{W}}{T_{f}-T_{W}}, \quad y_{F}=\frac{Y_{F}}{Y_{F C}}, \quad y_{s}=\frac{Y_{s}}{Y_{F C}}, \\
& \xi=\frac{\rho v}{\rho_{u} v_{u}}, \quad z=\frac{\rho_{u} v_{u} C}{\lambda_{u}} x, \quad D=\frac{T_{f}-T_{W}}{T_{f}-T_{W}},
\end{aligned}
$$

in which $v_{u}$ is the burning velocity and $Y_{F C}$ is defined as:

$$
Y_{F C}=\frac{C}{Q}\left(T_{f}-T_{u}\right) .
$$

Introducing the aforementioned parameters into Eqs. (4) and (5) results in:

$$
\begin{aligned}
& \xi \frac{d y_{s}}{d z}=-D \gamma \sqrt[3]{y_{s}^{2}} \theta^{n} \\
& \xi \frac{d y_{F}}{d z}=\frac{1}{L e} \frac{d^{2} y_{F}}{d z^{2}}-\omega D \frac{\rho_{u}}{\rho}+D \gamma \sqrt[3]{y_{s}^{2}} \theta^{n} \\
& \xi \frac{d \theta}{d z}=\frac{d^{2} \theta}{d z^{2}}+\omega \frac{\rho_{u}}{\rho}-q \gamma \sqrt[3]{y_{s}^{2}} \theta^{n}-k \theta \operatorname{sign}(z)
\end{aligned}
$$

where:

$$
\begin{aligned}
\omega & =\frac{\lambda_{u} \omega_{F}}{D \rho_{u} v_{u}^{2} C Y_{F C}}, \\
\gamma & =4.836\left(\sqrt[3]{\frac{n_{s}}{\rho_{u}^{4} \rho_{s}^{2} Y_{F C}}}\right) \frac{A \lambda_{u}\left(T_{f}-T_{u}\right)}{v_{u}^{2} C D}, \\
q & =\frac{Q_{v}}{Q}, \quad k=\frac{h H P e^{-2}}{\lambda_{u}}, \quad \text { Le }=\frac{\lambda_{u}}{\rho_{u} C D_{u}} .
\end{aligned}
$$

The heat recirculation coefficient is shown by $k$ in the above equation. In order to calculate the combustion parameters, the non-dimensional differential equations should be solved for all zones. Note that the quantity $m$ can be considered as unity in solving these equations.

\subsection{Preheat zone $\left\{x \mid-\infty<x<0^{-}\right\}$}

To solve the above equations, it is necessary to apply the boundary conditions. The required boundary conditions for solving conservation equations in this zone are as follows:

$$
\begin{aligned}
& z=-\infty \rightarrow\left\{\begin{array}{l}
\theta^{0}=0 \\
y_{s}=\frac{Y_{F u}}{Y_{F} C}=\alpha \\
y_{F}=\frac{d y_{F}}{d z}
\end{array}\right. \\
& z=0 \rightarrow\left\{\begin{array}{l}
\theta^{0}=1 \\
y_{F}=y_{F f}
\end{array}\right.
\end{aligned}
$$


The Zeldovich number is considered large enough in this zone. Therefore, it is possible to disregard the chemical reaction between the fuel and oxidizer. The amount of heat released by combustion is much larger than the heat needed for vaporization of solid particles $(q=0)$. The following correlations can be achieved by incorporating the above assumptions and using the required boundary condition:

$$
\begin{aligned}
& \theta^{0}=\exp \left(k_{1} z\right), \quad k_{1}=0.5+0.5(1-4 k)^{0.5} \\
& y_{s}=\left(\alpha^{\frac{1}{3}}-a \exp \left(n k_{1} z\right)\right)^{3}, \quad a=\frac{\gamma D}{3 n k_{1}} \\
& -\frac{d y_{F}}{d z} \mid\left(z=0^{-}\right)=\operatorname{Le}\left(3 \exp (-\mathrm{Bi}) a \alpha^{\frac{2}{3}}\right. \\
& \left.-3 \exp (-2 \mathrm{Bi}) a^{2} \alpha^{\frac{1}{3}}+\exp (-3 \mathrm{Bi}) a^{3}-y_{F f}\right)
\end{aligned}
$$

In these equations, $\theta^{\circ}$ represent the value of $\theta$ calculated by neglecting heat of vaporization of particles.

2.2. Post-flame zone $\left\{x \mid 0^{+}<x<+\infty\right\}$

Solving the governing equations in this zone provides the appropriate boundary conditions for the second zone (reaction zone). As mentioned previously, the diffusive term can be omitted in this zone. This assumption leads to the correlations below:

$$
\begin{aligned}
& \frac{d \theta^{0}}{d z}=-k \theta^{0} \rightarrow{\left.\frac{d \theta^{0}}{d z}\right|_{\left(z=0^{+}\right)}}=-k, \\
& \frac{d y_{F}}{d z}=0 \rightarrow{\frac{d y_{F}}{d z_{\mid}\left(z=0^{+}\right)}}=0, \\
& \frac{d y_{s}}{d z}=0 \rightarrow y_{s \mid\left(z=0^{+}\right)}=\text {const. }
\end{aligned}
$$

\subsection{Reaction zone $\left\{x \mid 0^{-}<x<0^{+}\right\}$}

In the reaction zone, the convective and vaporization terms can be ignored. The thickness of this zone is so small. Therefore, an expansion procedure is employed to solve the related equation. To this aim, the following parameters are used to obtain the differential equations:

$$
z=\varepsilon \eta, \quad y_{F}=\varepsilon(b+y), \quad \theta^{0}=1-\varepsilon t,
$$

where $b=\frac{y_{F f}}{\varepsilon}$ and $\varepsilon=\frac{1}{Z e}$. Assuming a large Zeldovich number, $\varepsilon$ will be too small. Introducing the mentioned parameters into Eq. (21) associated with the stated assumptions yields:

$$
\begin{aligned}
& \frac{d^{2} t}{d \eta^{2}}=\varepsilon \omega \frac{\rho_{u}}{\rho}, \\
& \frac{d^{2} y}{d \eta^{2}}=\varepsilon \omega D L e \frac{\rho_{u}}{\rho} .
\end{aligned}
$$

Introducing $\omega$ from Eq. (15) into the above equation results in:

$$
\frac{d^{2} t}{d \eta^{2}}=\Lambda(b+y) \exp (-t)
$$

where:

$$
\Lambda=\frac{\lambda_{u} v_{F} B \varepsilon^{2}}{D \rho_{u} v_{u}^{2} C} \exp \left(-\frac{E_{a}}{R T_{f}}\right) .
$$

By combining Eqs. (23) and (24), one can obtain:

$$
\frac{d^{2}(y-\mathrm{Le} D t)}{d \eta^{2}}=0 \text {. }
$$

The required boundary conditions are provided below:

$$
\begin{aligned}
& {\left[\frac{d t}{d \eta}\right]_{\eta \rightarrow \infty}=-\left[\frac{d \theta^{0}}{d z}\right]_{z \rightarrow 0^{+}}=k,} \\
& {\left[\frac{d y}{d \eta}\right]_{\eta \rightarrow \infty}=-\left[\frac{d y_{F}}{d z}\right]_{z \rightarrow 0^{+}}=0,} \\
& {\left[\frac{d t}{d \eta}\right]_{\eta \rightarrow-\infty}=-\left[\frac{d \theta^{0}}{d z}\right]_{z \rightarrow 0^{-}}=k_{1} .}
\end{aligned}
$$

Solving Eq. (25) leads to the following expression:

$$
y=\mathrm{Le} D t \text {. }
$$

Finally, by using this expression and integrating the above equations, we have:

$$
2 \alpha \Lambda(b+\operatorname{Le} D)=k_{1}^{2}+k^{2} .
$$

\subsection{Flame temperature and burning velocity}

The analytical expression for flame speed (burning velocity) is given by:

$$
v_{u}^{2}=\frac{2(b+\operatorname{Le} D) \lambda_{u} \nu_{F} B \varepsilon^{2}}{D \rho_{u} C\left(k_{1}^{2}+k^{2}\right)} \exp \left(-\frac{E_{a}}{R T_{f}}\right) .
$$

Using the matching condition in the border of the reaction zone can provide the flame temperature as:

$$
\left[\frac{d \theta^{0}}{d \xi}+\frac{1}{\mathrm{Le}} \frac{d y_{F}}{d \xi}\right]_{\xi \rightarrow 0^{+}}=\left[\frac{d \theta^{0}}{d \xi}+\frac{1}{\mathrm{Le}} \frac{d y_{F}}{d \xi}\right]_{\xi \rightarrow 0^{-}}
$$

Hence:

$$
\begin{aligned}
\left(3 \exp (-\mathrm{Bi}) a \alpha^{\frac{2}{3}}-3 \exp (-2 \mathrm{Bi}) a^{2} \alpha^{\frac{1}{3}}\right. \\
\left.+\exp (-3 \mathrm{Bi}) a^{3}+k+k_{1}\right)=0 .
\end{aligned}
$$

Although the above expression obtains the flame temperature, its value must be limited by the adiabatic temperature (considering that the oxidizer is consumed at the end of the process). The following expression calculates the adiabatic temperature:

$$
C\left(T_{b}-T_{u}\right)=\frac{v_{F} W_{F} Q}{v_{O_{2}} W_{O_{2}}} Y_{O_{2}, u}
$$

Until now, the whole solution procedure has neglected the parameter $q$. Considering this parameter alters the burning velocity in the following formula:

$$
v_{v}=v_{u} \exp (-0.5(q Z e)) \text {. }
$$




\section{Random model}

Since the dispersion of particles is not uniform in the flame front, the combustion properties including flame temperature, flame speed, and the amount of released energy are subject to change in this region. The released energy in the reaction zone, controlled by the source term in the energy equation, is a function of net available fuel in gaseous phase that varies in the flame front because of the random behavior of the fuel. The amount of this energy is dependent on the following parameters:

- Size. It has been found that the fuel particles with different sizes and shapes directly affect the combustion performance of the process. Here, it is assumed that the fuel particles are mono-sized [20];

- Number density. This parameter is explained as the total number of particles per unit volume by:

$$
n_{s}=\frac{N_{s}}{V}
$$

The equivalence ratio is calculated by:

$$
\begin{aligned}
\phi_{u} & =\left(\frac{Y_{F u}}{Y_{a i r}}\right) /\left(\frac{Y_{F u}}{Y_{a i r}}\right)_{s t} \\
& =\frac{Y_{F u}}{1-Y_{F u}}\left(\frac{\nu_{F} W_{F}}{\nu_{a i r} W_{a i r}}\right)_{s t}^{-1},
\end{aligned}
$$

At the beginning of the preheat zone, the parameters $Y_{F u}$ and $Y_{s}$ are equal. Therefore, the equivalence ratio is given by:

$$
\phi_{u}=\frac{Y_{s}}{1-Y_{s}}\left(\frac{\nu_{F} W_{F}}{\nu_{a i r} W_{a i r}}\right)_{s t}^{-1},
$$

where $Y_{s}$ is obtained by Eq. (37):

$$
Y_{s}=\left(\frac{4}{3} \pi r^{3}\right) \frac{n_{s} \rho_{s}}{\rho} .
$$

It is assumed that the fuel particles are volatilized to form methane [21]. Hence:

$$
\left(\frac{\nu_{F} W_{F}}{\nu_{a i r} W_{a i r}}\right)_{s t}^{-1}=17.12 .
$$

Hence:

$$
n_{s}=\left(\frac{4}{3} \pi r^{3}\right)^{-1} \frac{\rho \phi_{u}}{\rho_{s}\left(17.12+\phi_{u}\right)} .
$$

In an ideal case, not only the dispersion of these particles in the flame front is uniform, but also the condition of these particles is the same in the preheat zone. However, none of these hypotheses can be applied to the real case. Since the temperature changes from

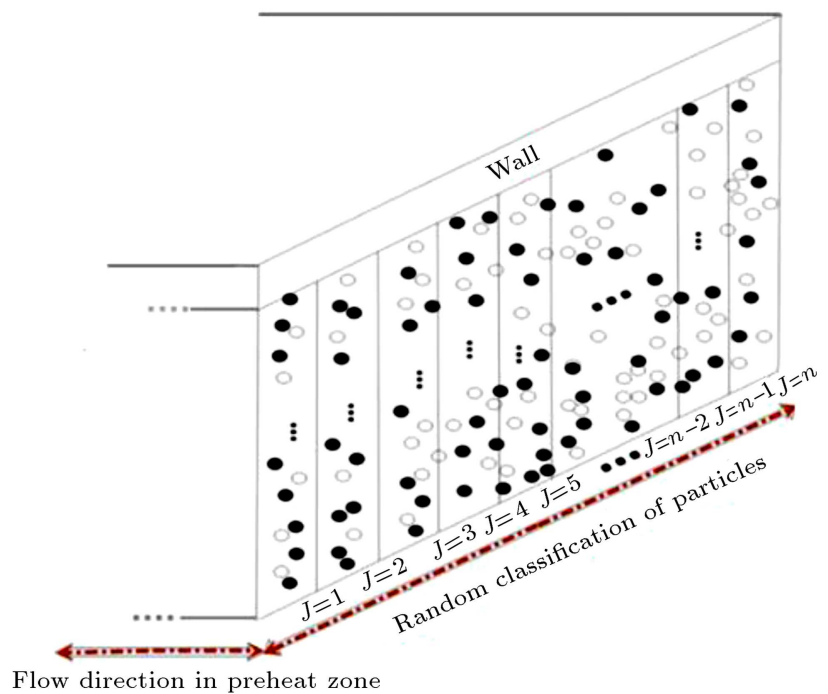

Figure 2. Schematic view of random distribution of particles in the preheat vaporization zone.

ambient to flame in the preheat zone, particles are subject to various temperatures determined by the gradient of temperature. Consequently, the amount of volatilized fuel for each particle would be remarkably different. Figure 2 shows schematics of random distribution of particles (in the preheat-vaporization zone).

In order to model the random distribution of the particles in the flame front, the source term in the energy equation needs to be amended. For this purpose, several groups of particles are assumed each containing a random amount of particles with a random temperature between flame and ambient temperatures in the preheat zone. The total available fuel is calculated as the sum of volatilized fuels from all groups as shown in Eqs. (40) and (41) [22]:

$$
\begin{aligned}
n_{s, t} & =\sum_{j=1}^{g} n_{s_{j}}, \\
\omega_{v, t} & =\sum_{j=1}^{g} \omega_{v_{j}}=\sum_{j=1}^{g} 4 \pi r^{2} A n_{s_{j}} T_{j}^{n}, \\
& T_{u}<T_{j}<T_{f} .
\end{aligned}
$$

The reaction rate is obtained from the following equation:

$$
\omega_{f}=\sum_{j=1}^{g} \omega_{f_{j}}=v_{f} k_{r} \sum_{j=1}^{g} \omega_{v_{j}} .
$$

Additionally, the source or generation term is formulated as:

$$
E_{g e n}=\sum_{j=1}^{g} \frac{\rho_{u}}{\rho} Q \omega_{f_{j}},
$$

where parameter $g$ signifies the number of particle 
groups. The number density for all groups varies from zero to $n_{s t}$. In uniform distribution of particles, all particles have identical behaviors. Therefore, there is one group for all particles with the number density of $n_{s t}$. Nevertheless, the conditions of various groups of particles would vary with the assumption of randomness.

The aim of this analysis is to estimate the effects of Lewis number, heat recirculation, and random distribution of particles with variation in wall temperature on the combustion behavior and flame structure of the organic dust particles.

\section{Results and discussion}

In this study, the physical geometry of the domain is determined by an infinitely open tube with the width of $H$. Another advantage of this research lies in addressing the influence of the resistance of particles thermal on the form of Biot number. At the beginning, it is presumed that the organic dust particles will be volatilized to form methane as the gaseous fuel. Methane is then combusted in the reaction zone.

Basically, vaporization rate and the amount of released gaseous fuel are contingent on the temperature of particles in the preheat zone. Temperature distribution was analytically investigated versus the length of the preheat zone, implying that particle distribution would strongly affect the vaporization rate. This important finding is taken into account by dividing the particles into different groups in modeling their random dispersal. The heat capacity and adiabatic flame temperature are calculated as functions of equivalence ratio. Finally, analytical expression is derived for burning velocity and flame temperature.

Assessing the accuracy of the model is done by comparing the burning velocity obtained from the present model with the experimental findings of Proust [11] and Han et al. [20] as well as the analytical data of Seshadri et al. [21]. As displayed in Figure 3, both random and Seshadri non-random models have a practical compatibility with experimental data. Nonetheless, the random model has a better estimation. The achieved results in this research reveal that the random model leads to a better prediction of empirical burning velocity in comparison with the obtained data for uniform condition from Seshadri's model $[11,20,21]$. Moreover, it is clear that wall temperature and thermal resistance have a significant impact on the calculated burning velocity by the presented mathematical approach. Seshadri's theoretical work is based on the assumption that wall temperature is constant and equal to the ambient temperature.

However, it increases due to heat transfer to the wall from the reaction products.

As shown in Figure 4, an increase in Biot number

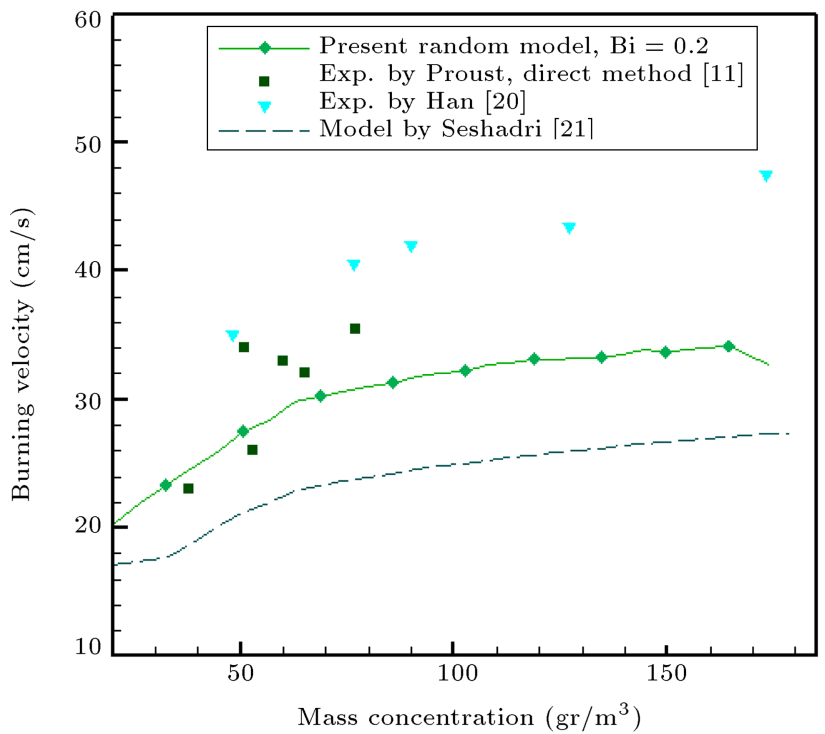

Figure 3. Variation of burning velocity as a function of mass concentration in the present model, experimental data $[11,20]$ and analytical model [21].

decreases the burning velocity and flame temperature, while it leads to an increase in thermal resistance of particles in varied temperature layers and pyrolysis rates inside the particles. The main reason for this behavior can be the reduction in the amount of produced gaseous fuel. This will result in lower reaction rate and consequently, lower flame speed and temperature. According to Figure 4, as the equivalence ratio grows, the flame temperature and burning velocity increase. In fact, pyrolysis resistance decreases as the mass particle density is reduced.

Figure 5 exhibits the flame temperature versus equivalence ratio for both uniform and random models. As seen, the maximum flame temperature cannot exceed the adiabatic flame temperature, which is why there is a threshold for the equivalence ratio at each radius of the particles. As observed in this figure, the flame temperature increases with the rise of the equivalence ratio. High equivalence ratio leads to higher amounts of reaction heat and, as a result, higher flame temperature. Another observation from the above figure pertains to the role of particle radius, indicating that a reduction in particle radius enhances the flame temperature as the two-phase mixture approaches the purely gaseous combustible mixture. It is revealed that there is a substantial difference between the uniform and random data at a fixed equivalence ratio. Although the random model has a considerably greater flame temperature than the uniform model for the smallest particle size, this gap gradually decreases for larger particles and, at some stages, the flame temperature achieved by the uniform model outweighs the one by the random model. The reason is the lower energy needed for vaporizing the smaller particles and 


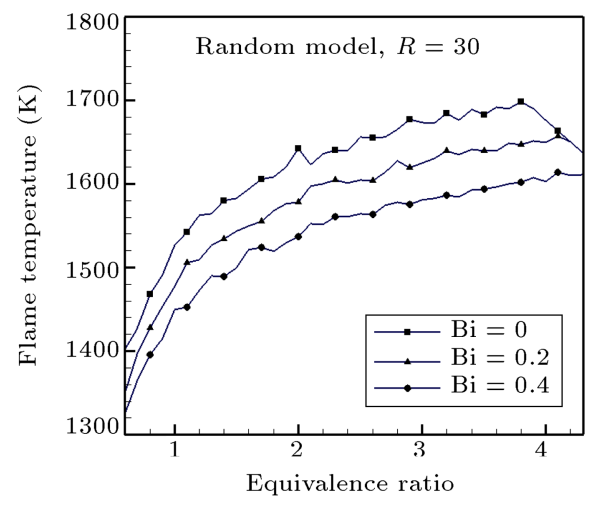

(a)

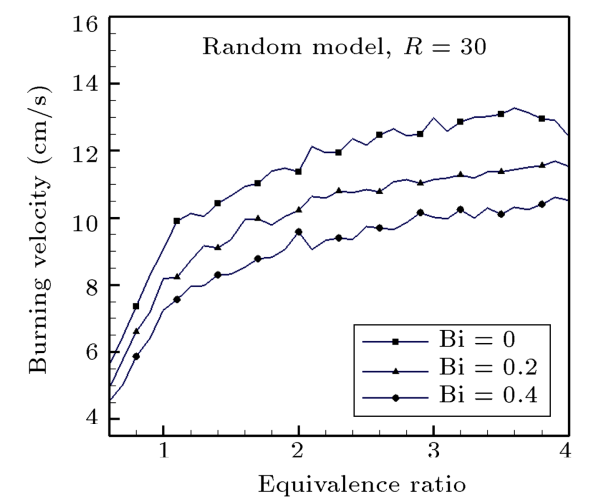

(b)

Figure 4. Variation of (a) flame temperature and (b) burning velocity as a function of equivalence ratio for different Biot numbers.

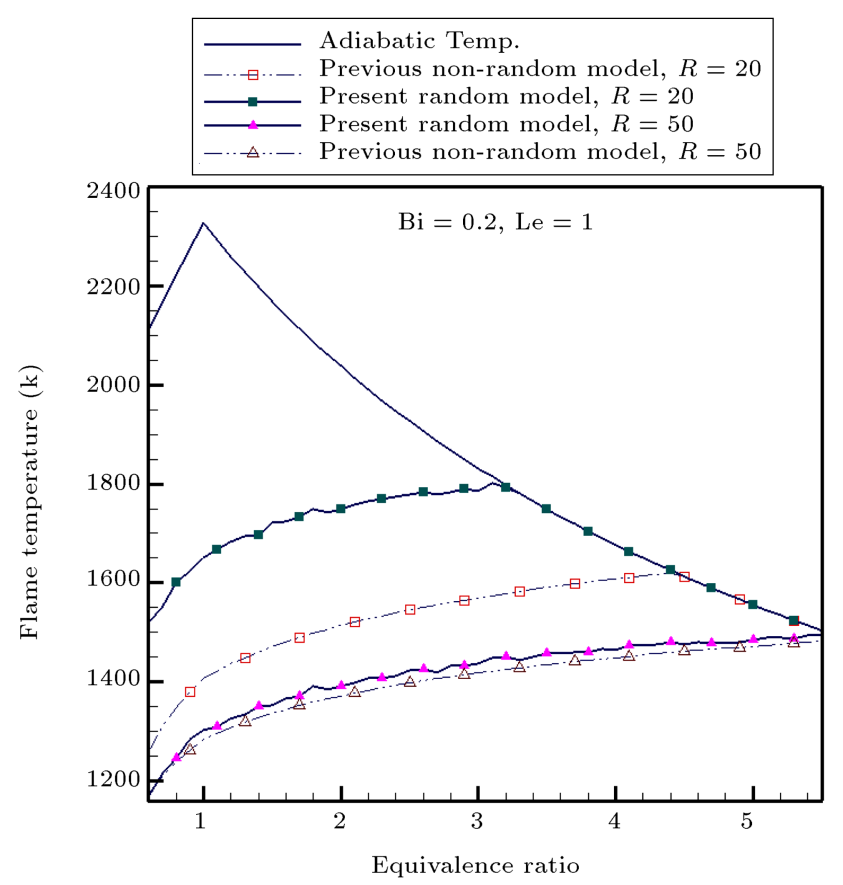

Figure 5. Variation of flame temperature versus equivalence ratio for both uniform and random models with respect to different particle radii at $\mathrm{Bi}=0.2$ and Le $=1$.

the higher number densities for smaller particles at a constant equivalence ratio. Thus, it is more likely for particles to initiate the vaporization process in the preheat zone. However, at higher particle radii, this probability significantly decreases.

Figure 6 illustrates the change in burning velocity as a function of the equivalence ratio for various radii of the particles. Apparently, the enhancement in the equivalence ratio is associated with the growth in the flame speed. Additionally, the burning velocity declines as the particle radius rises. The same reasoning as that for the flame temperature can be applied to burning velocity to justify the trend in this figure. As mentioned earlier, the random model demonstrates

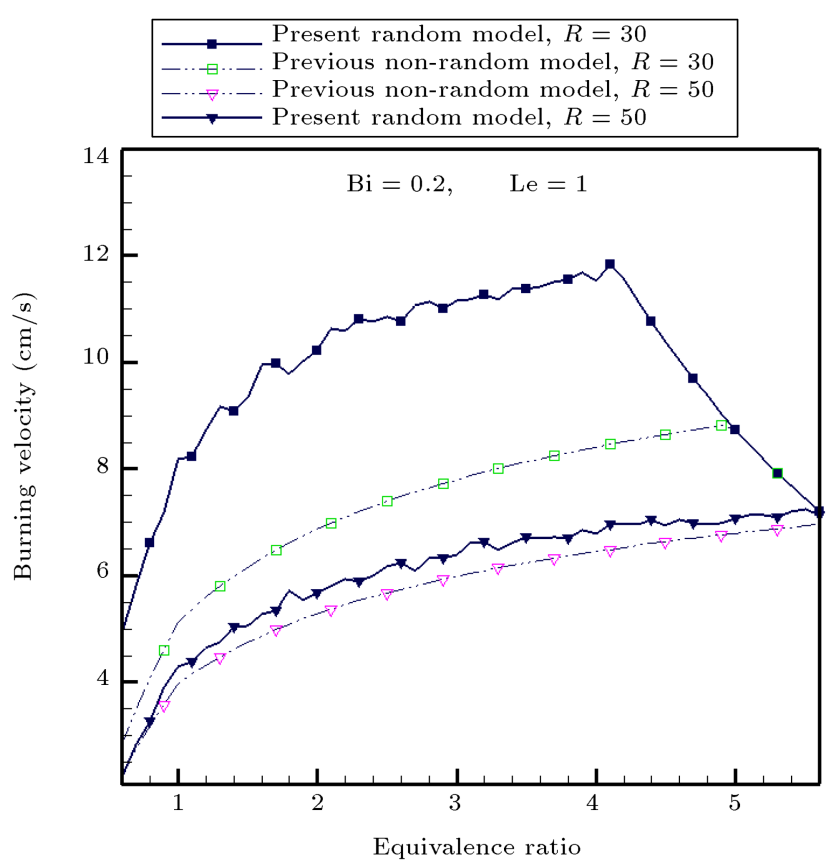

Figure 6. Variation of burning velocity versus equivalence ratio for both uniform and random models with respect to different particle radii at $\mathrm{Bi}=0.2$ and $\mathrm{Le}=1$.

lower amounts of flame speed for bigger particles and vice versa in comparison with the non-random mode.

According to Figures 5 and 6 , there is a reduction in the burning velocity and flame temperature at certain quantities of equivalence ratio for various radii of particles. This is a consequence of the following events. Initially, the preheat and flame temperatures increase with the rise of equivalence ratio and released heat of reaction. Gradually, the quantity of vaporized fuel becomes equivalent to the stoichiometric value. At this situation, flame temperature and the effective equivalence ratio are identical to unity and adiabatic temperature, respectively. On the other hand, with an increase in the equivalence ratio, the effective equivalence ratio will remain stable. This implies that 


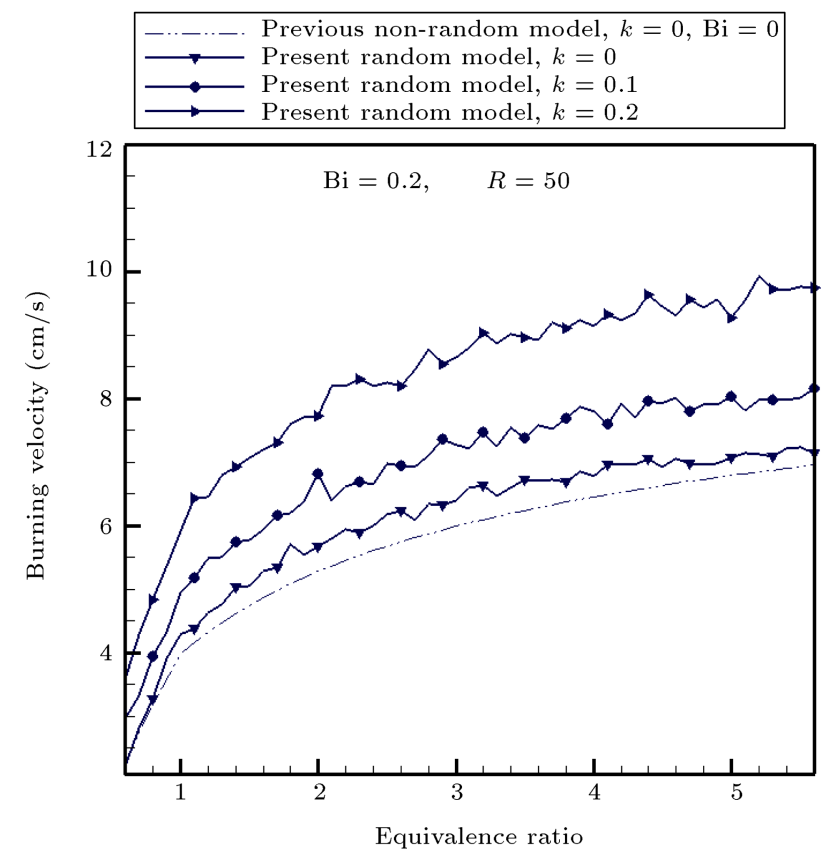

Figure 7. Variation of burning velocity versus equivalence ratio for different heat recirculation coefficients $(k)$ at $\mathrm{Bi}=0.2$ and $r_{u}=50 \mu \mathrm{m}$.

the heat of reaction does not vary while more reactants participate in the reaction process. Therefore, the flame speed and temperature start decreasing above the specific equivalence ratio (effective equivalence ratio becomes equal to unity).

Heat recirculation is one of the dominating parameters in the combustion performance of biofuels. Figure 7 demonstrates this for burning velocity with $r_{u}=50 \mu \mathrm{m}$. As shown, there is a direct relationship between these two parameters. The flame temperature and flame speed go up with the heat recirculation coefficient. The reason is that recirculating the heat from post-flame to the preheat zone enhances the temperature of reactants. Therefore, reactants require less energy for combustion process and the excess energy from the exothermic reaction further increases the flame temperature and, as a consequence, flame speed.

Figure 8 illustrates the variation of flame speed with equivalence ratio for different values of $D$, $\left(D=\left(T_{f}-T_{w}\right) /\left(T_{f}-T_{u}\right)\right)$. In Figure 8, flame temperature and burning velocity rise with increase in the wall temperature or decrease in the parameter $D$. The wall temperature is proportional to the amount of heat recirculation. This is possible under two different mechanisms:

(a) The energy can be conducted through the wall and then, absorbed by the mixture in the preheat zone via convective heat transfer;

(b) The geometry of the combustion chamber is de-

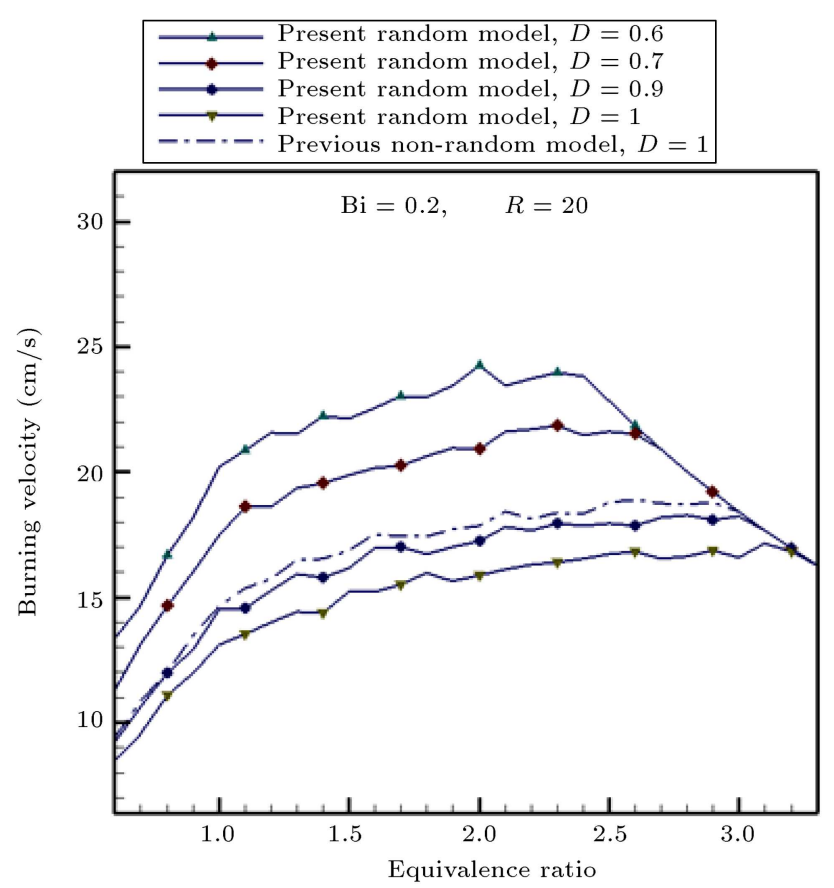

Figure 8. Variation of the burning velocity versus equivalence ratio for different values of $D$ at $\mathrm{Bi}=0.2$ and $r=20 \mu \mathrm{m}$.

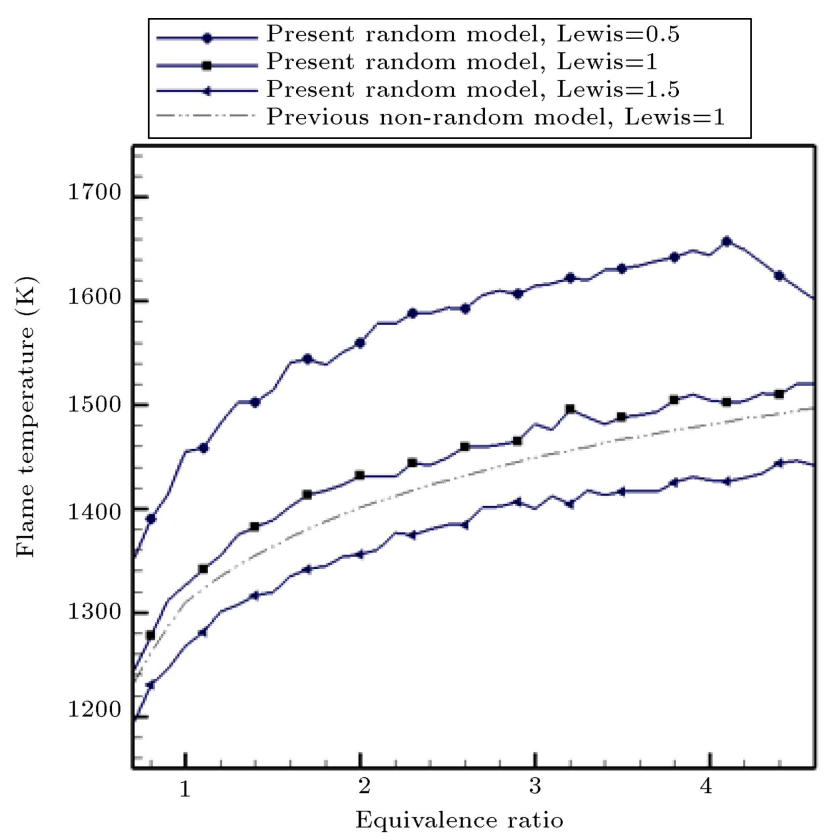

Figure 9. Variation of the flame temperature versus equivalence ratio for different Lewis numbers.

signed in a way that the exhausted flow in the post-flame zone enters a tube and goes back to the preheat zone in the opposite direction of fluid flow.

In both methods, the temperature of wall as well as convective heat transfer coefficient exerts a significant effect on the amount of recirculation.

Figure 9 indicates flame temperature as a function 
of equivalence ratio for various Lewis numbers. As observed, there is a reduction in flame temperature with increase in the Lewis number due to a rise in thermal diffusivity per mass diffusivity. This denotes that a lower amount of heat is transferred to the combustion flow. Consequently, the flame temperature decreases with a growth in the Lewis number.

\section{Conclusion}

This research investigated the flame structure of organic dust particles considering random distribution and thermal resistance of particles. The accuracy of the model was checked against corresponding experimental and analytical data. It was observed that the random model predicted the experimental findings published in the literature better than the uniform mode. After validation of the model, the impact of significant parameters including Biot number, heat recirculation, wall temperature, and Lewis number on the combustion properties was investigated. It was concluded that when the Biot number increased, different thermal layers with various pyrolysis rates formed inside the particles and consequently, less amount of gaseous fuel was produced during the pyrolysis procedure. The amount of heat recirculation approached the maximum by taking the wall temperature. Therefore, the amount of vaporization in the preheat zone, flame temperature, and burning velocity of the particles increased. It was observed that the equivalence ratio approached the stoichiometric condition at lower quantities with enhancement in the wall temperature. Afterwards, the combustion properties were the same as those for the adiabatic condition. It was also found that a rise in parameter $k$ would lead to a growth in both flame speed and flame temperature. The rise in the Lewis number was accompanied by a reduction in the flame temperature. The flame temperature and flame speed increased with the equivalence ratio because of the reduction in the pyrolysis resistance. Consequently, smaller particles possessed higher amounts of the produced fuel mixture, which had a tendency to increase the flame temperature and burning velocity, dramatically.

\section{Nomenclature}

A Rate of vaporization of fuel particles

$a \quad$ Defined in Eq. (18)

$B \quad$ Pre-exponential factor of the reaction

Bi Biot number

$C \quad$ Heat capacity of the mixture

$C_{P} \quad$ Heat capacity of the gas

$C_{s} \quad$ Heat capacity of a fuel particle
$D \quad$ Dimensionless flame temperature

$E_{a} \quad$ Activation energy of the reaction

$H \quad$ Chamber width

$h \quad$ Convective heat transfer coefficient

$k \quad$ Heat recirculation coefficient defined in Eq. (15)

$k_{r} \quad$ Constant rate of the gas-phase reaction

$k_{1} \quad$ Defined in Eq. (17)

$L \quad$ Thickness of the thermal mixing layer

Le Lewis number

Pe Peclet number

$Q \quad$ Heat release per unit mass of the fuel burned

$q \quad$ Defined in Eq. (15)

$Q_{v} \quad$ The heat associated with the vaporizing unit mass of fuel

$R \quad$ Gas constant

$r \quad$ Radius of fuel particle

$\nu_{u} \quad$ Burning velocity calculated neglecting heat of vaporization of fuel particles

$\nu_{v} \quad$ Burning velocity calculated including heat of vaporization of fuel particles

$Y_{F} \quad$ Mass fraction of the gaseous fuel

$Y_{F C} \quad$ Defined in Eq. (11)

$y_{F} \quad$ Dimensionless mass fraction of the gaseous fuel

$Y_{s} \quad$ Mass fraction of particles

$y_{s} \quad$ Dimensionless mass fraction of particles

$n \quad$ Temperature exponent characterizing rate of vaporization of fuel particles

$n_{u} \quad$ Number density of particles in the inlet stream

$n_{s} \quad$ Local number density of particles (number of particles per unit volume)

$W_{F} \quad$ Molecular weight of gaseous fuel

$\mathrm{Z}_{\mathrm{e}} \quad$ Zeldovich number

$z \quad$ Scaled independent variable defined in Eq. (10)

\section{Greek letters}

$\alpha \quad$ Defined in Eq. (16)

$\phi_{u} \quad$ Equivalence ratio based on the fuel available in the particles in the ambient reactant stream

$\omega \quad$ Defined in Eq. (15)

$\omega_{v} \quad$ Rate of vaporization of fuel particles

$\omega_{F} \quad$ Rate of chemical kinetic for fuel particles

$\gamma \quad$ Defined in Eq. (15)

$\lambda$ Thermal conductivity 
$\xi \quad$ Defined in Eq. (10)

$\Lambda \quad$ Defined in Eq. (24)

$\nu \quad$ Stoichiometric coefficient

$\rho \quad$ Density of the mixture

$\rho_{s} \quad$ Density of a fuel particle

$\varepsilon \quad$ Expansion parameter $(1 / \mathrm{Ze})$

$\eta \quad$ Independent variable defined in

Eq. (21)

$\theta \quad$ Dimensionless temperature of gas

$\theta^{\circ} \quad$ Value of $\theta$ calculated neglecting heat of vaporization of particles

\section{Subscripts}

$b \quad$ Adiabatic conditions after completion of chemical reactions

F $\quad$ Gaseous fuel

$f \quad$ Conditions in the flame

$s \quad$ Solid fuel

$u \quad$ Conditions in the inlet stream

\section{References}

1. Eckhoff, R.K., Dust Explosions in the Process Industries: Identification, Assessment and Control of Dust Hazards, Elsevier (2003).

2. Moghadasi, H., Rahbari, A., Bidabadi, M., Poorfar, A.K., and Farhangmehr, V. "A mathematical investigation of premixed lycopodium dust flame in a small furnace", Journal of Energy Resources Technology, 141(3), 032201 (2019).

3. Afzalabadi, A., Poorfar, A.K., Bidabadi, M., Moghadasi, H., Hochgreb, S., Rahbari, A., and Dubois, C. "Study on hybrid combustion of aero-suspensions of boron-aluminum powders in a quiescent reaction medium", Journal of Loss Prevention in the Process Industries, 49, pp. 645-651 (2017).

4. Moghadasi, H., Malekian, N., Bidabadi, M., and Rasam, H. "Analytical modeling of counterflow nonpremixed organic particles combustion: Thermal radiation effects", Fuel Processing Technology, 185, pp. 139-150 (2019).

5. Bidabadi, M., Xiong, Q., Harati, M., Yaghoubi, E., Doranehgard, M.H., and Rahbari, A. "Study on the combustion of micro organic dust particles in random media with considering effect of thermal resistance and temperature difference between gas and particles", Chemical Engineering and Processing-Process Intensification, 126, pp. 239-247 (2018).

6. Bidabadi, M., Aghajannezhad, P., Harati, M., Yaghoubi, E., and Shahriari, G. "Modeling random combustion of lycopodium particles and gas", International Journal of Spray and Combustion Dynamics, 8(2), pp. 100-111 (2016).

7. Bidabadi, M., Mostafavi, S.A., Dizaji, H.B., and Dizaji, F.F. "Lycopodium dust flame characteristics considering char yield", Scientia Iranica., Transactions B, Mechanical Engineering, 20(6), p. 1781 (2013).

8. Bidabadi, M., Panahifar, P., and Sadeghi, S. "Analytical development of a model for counter-flow nonpremixed flames with volatile biofuel particles considering drying and vaporization zones with finite thicknesses", Fuel, 231, pp. 172-186 (2018).

9. Bidabadi, M., Harati, M., Xiong, Q., Yaghoubi, E., Doranehgard, M.H., and Aghajannezhad, P. "Volatization \& combustion of biomass particles in random media: Mathematical modeling and analyze the effect of Lewis number", Chemical Engineering and ProcessingProcess Intensification, 126, pp. 232-238 (2018).

10. Mostafavi, S.A., Salavati, S., Dizaji, H.B., and Mehdi, B. "Pyrolysis and combustion kinetics of lycopodium particles in thermogravimetric analysis", Journal of Central South University, 22(9), pp. 3409-3417 (2015).

11. Proust, C. "A few fundamental aspects about ignition and flame propagation in dust clouds", Journal of Loss Prevention in the Process Industries, 19(2-3), pp. 104120 (2006).

12. Proust, C. "Flame propagation and combustion in some dust-air mixtures", Journal of Loss Prevention in the Process Industries, 19(1), pp. 89-100 (2006).

13. Han, O.S., Yashima, M., Matsuda, T., Matsui, H., Miyake, A., and Ogawa, T. "A study of flame propagation mechanisms in lycopodium dust clouds based on dust particles' behavior", Journal of Loss Prevention in the Process Industries, 14(3), pp. 153-160 (2001).

14. Daou, J. "Strained premixed flames: Effect of heatloss, preferential diffusion and reversibility of the reaction", Combustion Theory and Modelling, 15(4), pp. 437-454 (2011).

15. Bidabadi, M., Fanaee, A., and Rahbari, A. "Investigation over the recirculation influence on the combustion of micro organic dust particles", Applied Mathematics and Mechanics, 31(6), pp. 685-696 (2010).

16. Bidabadi, M. and Rahbari, A. "Modeling combustion of lycopodium particles by considering the temperature difference between the gas and the particles", Combustion, Explosion, and Shock Waves, 45(3), pp. 278-285 (2009).

17. Rockwell, S.R. and Rangwala, A.S. "Modeling of dust air flames", Fire Safety Journal, 59, pp. 22-29 (2013).

18. Moghadasi, H., Malekian, N., Poorfar, A.K., and Bidabadi, M. "Thermal radiative study of counterflow combustion of porous particles", Chemical Engineering and Processing-Process Intensification, 134, pp. 163173 (2018).

19. Soltaninejad, M., Dizaji, F.F., Dizaji, H.B., and Bidabadi, M. "Micro-organic dust combustion considering particles thermal resistance", Journal of Central South University, 22(7), pp. 2833-2840 (2015).

20. Han, O.S., Yashima, M., Matsuda, T., Matsui, H., Miyake, A., and Ogawa, T. "Behavior of flames propagating through lycopodium dust clouds in a vertical duct", Journal of Loss Prevention in the Process Industries, 13(6), pp. 449-457 (2000). 
21. Seshadri, K., Berlad, A.L., and Tangirala, V. "The structure of premixed particle-cloud flames", Combustion and Flame, 89(3-4), pp. 333-342 (1992).

22. Bidabadi, M., Harati, M., Afzalabadi, A., and Rahbari, A. "Effect of thermal resistance on the random combustion of micro-organic dust particles", Journal of Energy Engineering, 144(1), p. 04017073 (2017).

\section{Biographies}

Mehdi Bidabadi received his BSc degree in Mechanical Engineering from Iran University of Science and Technology, where he is now a faculty member, and MSc and $\mathrm{PhD}$ degrees from Sharif University of Technology, Iran, and McGill University, Canada, respectively. His research interests include dust flame propagation mechanisms, combustion, wind tunnels and gas dynamics, development of new experimental apparatuses to produce laminar flow, and optimization of smoke wind tunnels. He has published several research papers in the field of dust combustion.

Mohammadali Harati is $\mathrm{PhD}$ candidate in Mechanical Engineering at Iran University of Science and Technology, Iran. He received his BSc and MSc degrees from Sharif University of Technology, Iran. His $\mathrm{PhD}$ research involved the analytical study of random combustion of micro-organic and non-organic particles.

Hesam Moghadasi received his MSc degree in Mechanical Engineering at Iran University of Science and Technology (IUST), Iran, in 2017. He is currently a $\mathrm{PhD}$ candidate at IUST. His major research interests are fluid mechanics, boiling heat transfer, two-phase flow, CFD in applications, combustion, and analytical methods. He has published several research papers in the reputable international journals. 